\title{
Multiextrapolação de Richardson e Verificação da Ordem de Acurácia de Esquemas Híbridos sobre a Equação 2D de Fourier com Termo Fonte
}

\author{
Ana Paula da S. Vargas, \\ Faculdades Integradas do Brasil - Unibrasil \\ 82821-020, Curitiba, Paraná \\ Universidade Federal do Paraná - Departamento de Engenharia Mecânica \\ 81531-980, Curitiba, Paraná \\ E-mail: vargas.apaulas@gmail.com \\ Carlos H. Marchi, Marcio A. V. Pinto \\ Universidade Federal do Paraná - Departamento de Engenharia Mecânica \\ 81531-980, Curitiba, Paraná \\ E-mails: machi@pq.cnpq.br; marcio_villela@ufpr.br
}

\begin{abstract}
Resumo: Acurácia e previsões confiáveis em mecânica dos fluidos são os objetivos principais em CFD. O objetivo final de interesse científico é a validação de um modelo e para isso a verificação se faz necessária. Duas etapas da verificação são: a verificação do código e a verificação da solução. Em vista disso, este trabalho apresenta a solução da equação de Fourier 2D, com termo fonte e erro de truncamento combinando o método das soluções fabricadas (MSF) com a verificação da ordem de acurácia a priori e a posteriori. A estimativa a priori da ordem de acurácia é realizada por meio da dedução da ordem formal baseada na série de Taylor. Tal abordagem é utilizada com o objetivo de verificar efetivamente a ordem de acurácia das soluções numéricas calculada a posteriori pela utilização de técnicas hibridas. A estimativa a posteriori é realizada com base na estimativa do erro de discretização obtida por meio de Multiextrapolações de Richardson (MER). As soluções são obtidas com o emprego dos Métodos de Diferenças Finitas (MDF) e multigrid. Os resultados obtidos indicam que: i) ofator de mistura não altera a eficácia de MER; ii) a ordem de acurácia obtida a posteriori por meio de MER corrobora a ordem formal obtida a priori; iii) MER provê subsídios para os casos em que não se consegue estimar a priori ou a posteriori as ordens do erro numérico. Tópicos adicionais incluem a dedução das ordens verdadeiras do esquema numérico, análise da ordem aparente, efeito de parâmetro numérico em esquema híbrido e equação parabólica com termo fonte.
\end{abstract}

Palavras-chave: Multiextrapolação de Richardson, termo fonte, ordem de acurácia, método das soluções fabricadas, verificação numérica, CFD, equação de Fourier 2D, esquemas híbridos.

\section{Introdução}

A necessidade de predizer com acurácia resultados numéricos que representem o comportamento de fluidos em movimento é de grande importância em CFD (Computational Fluid Dynamics). Avanços têm sido observados em estudos e análises no desenvolvimento de sistemas complexos com difícil aferição de soluções analíticas e/ou numéricas com base em subsídios fornecidos nesta área.

Apesar disso, controvérsias existentes na literatura a respeito de esquemas mistos devem ser esclarecidas como, por exemplo, que a ordem assintótica de um esquema híbrido (ou misto) é variável [1]. Em [8] sugere-se usar a menor ordem entre dois esquemas puros. Há relatos que, mesmo em problemas unidimensionais, o erro se reduz de forma não-monotônica quando se usa pelo menos dois esquemas com ordens assintóticas diferentes [9].

É comum encontrar em trabalhos científicos de diversas áreas, especificamente em CFD, várias combinações de métodos de solução. O interesse em esquemas híbridos está em determinar um modelo numérico apropriado de forma a atender altos níveis de acurácia. Nesse sentido, a verificação da ordem de acurácia da solução numérica versus ordem de acurácia 
formal da técnica numérica tem como objetivo identificar em que medida um modelo numérico é resolvido adequadamente. Para tanto, dois métodos são abordados: estimativa de erro a priori e estimativa de erro a posteriori [6].

A estimativa a priori da ordem de acurácia é realizada por meio da dedução da ordem formal baseada na série de Taylor. Tal abordagem é utilizada com o objetivo de verificar efetivamente a ordem de acurácia das soluções numéricas calculada a posteriori pela utilização de técnicas híbridas.

Para estimar, a posteriori, a magnitude da ordem do erro de discretização bem como a influência do parâmetro numérico de esquemas híbridos emprega-se o método de extrapolação de Richardson (ER) [7] de forma recursiva. Esse procedimento é conhecido por Multiextrapolação de Richardson (MER) (ou Repeated Richardson Extrapolation - RRE) [5]. ER também é bastante utilizada para reduzir o erro de discretização e aumentar a ordem de acurácia, pois cada aplicação representa um nível de extrapolação, a sua eficácia pode ser potencializada.

O objetivo final de interesse científico é a validação de um modelo e para isso a verificação se faz necessária $[6,10]$. Duas etapas da verificação são a verificação do código e a verificação da solução. A verificação do código examina se o algoritmo foi corretamente implementado $[3,10]$, e para isso MSF é recomendado. A verificação da solução é usada para estimar os erros numéricos que ocorrem na simulação numérica [6]. O uso de ER, nesse caso, é indicado.

Com isso, os objetivos deste trabalho são (1) análise da ordem de acurácia da solução numérica versus ordem de acurácia formal da técnica numérica; (2) verificação do efeito do parâmetro numérico na solução e no erro numérico; (3) verificação da solução com o emprego de MER.

\section{Conceitos e métodos}

$\mathrm{O}$ erro da solução numérica, ou simplesmente erro numérico $(E)$, é a diferença entre a solução analítica exata $(\Phi)$ de uma variável de interesse e sua solução numérica $(\phi)$ [4], ou seja,

$$
E(\phi)=\Phi-\phi
$$

O erro numérico é causado basicamente por quatro fontes, que são: erros de iteração, erros de truncamento, erros de arredondamento e erros de programação [4]. Quando as demais fontes são minimizadas ou inexistentes, o erro de truncamento passa a ser denominado erro de discretização $(E h)$. Similarmente à forma geral do erro de truncamento, Eh é dado por

$$
E h=c_{1} h^{p_{L}}+c_{2} h^{p_{2}}+c_{3} h^{p_{3}}+\cdots \quad \text { para } h \rightarrow 0
$$

onde o menor expoente de $h$ na expressão de $E h$ na Eq. (2), $p_{L}$, chamamos ordem assintótica ou de acurácia de $E h$. A ordem assintótica $\left(p_{L}\right)$ e os expoentes dos termos não nulos na equação do erro de truncamento $\left(p_{2}, p_{3}, \ldots\right)$ são denominadas ordens verdadeiras $\left(p_{V}\right)$. O conjunto representado por $p_{V}$ e seus elementos são números reais e seguem a relação: $1 \leq p_{L}<p_{2}<$ $p_{3}<\cdots$ Todas as ordens verdadeiras são valores conhecidos. Os coeficientes reais $c_{1}, c_{2}, c_{3}, \ldots$ independem de $h$ e podem ser positivos ou negativos e podem ser função da variável dependente e de suas derivadas. Tanto os coeficientes $c_{i}$ como as ordens verdadeiras dependem das aproximações numéricas aplicadas.

A ideia básica de ER exige a solução numérica da variável de interesse em duas malhas com diferentes números de nós. Por exemplo, com base nas Eqs. (1) e (2), consideram-se as soluções aproximadas $\phi(h)$ e $\phi(r h)$ representadas por

$$
\begin{gathered}
\Phi-\phi(h)=c_{1} h^{p_{L}}+\mathcal{O}\left(h^{p_{2}}\right) \\
\Phi-\phi(r h)=c_{1}(r h)^{p_{L}}+\mathcal{O}\left(h^{p_{2}}\right)
\end{gathered}
$$

Subtraindo a Eq. (4) da Eq. (3), obtém-se a expressão para o erro de truncamento: 


$$
c_{1} h^{p_{L}}=\frac{\phi(h)-\phi(r h)}{r^{p_{L}}-1}+\mathcal{O}\left(h^{p_{2}}\right)
$$

Assim, substituindo a Eq. (5) na Eq. (3) chega-se a

$$
\Phi=\phi(h)+\frac{\phi(h)-\phi(r h)}{r^{p_{L}}-1}+\mathcal{O}\left(h^{p_{2}}\right)
$$

A aplicação repetida da Eq. (6) é denominada por Multiextrapolação de Richardson (MER) [5] em que a primeira aplicação é considerada o primeiro nível de extrapolação, chegando-se a $p_{L}$. Com a aplicação sucessiva desse processo, definem-se os valores sequenciais das ordens por $p_{m}$, cada nível de malha por $g$ e $m$ o número de aplicações de ER pode-se reescrever a Eq. (6) como

$$
\phi_{g, m}=\phi_{g, m-1}+\frac{\phi_{g, m-1}-\phi_{g-1, m-1}}{r^{p_{m-1}-1}}
$$

em que $g=2, \ldots, G, r=h_{g-1} / h_{g}$ e $m=1, \ldots, g-1$; e $\phi_{g, 0}$ representa $\phi$ sem o emprego de ER. Teoricamente, quanto maior o valor de $m$, mais próximo $\phi_{g, m}$ estará de $\Phi$. O valor máximo de $m$ permitido é $m=1, \ldots, M$ onde $M=g-1$. A acurácia de $\phi_{M}$ pode ser analisada com base nos conceitos de ordem efetiva $\left(p_{E}\right)$ e ordem aparente $\left(p_{U}\right)$, isto é,

$$
p_{E g, m}=\frac{\log \left[\frac{\Phi-\phi_{g-1, m-1}}{\Phi-\phi_{g, m}}\right]}{\log (r)} \quad e \quad p_{U_{g, m}}=\frac{\log \left[\frac{\phi_{g-1, m-1}-\phi_{g-2, m-2}}{\phi_{g, m}-\phi_{g-1, m-1}}\right]}{\log (r)}
$$

assumindo-se $g=2, \ldots, G$ para $p_{E g, m}, g=3, \ldots, G$ para $p_{U g, m}$ e $m=1, \ldots, M$.

\section{Modelo Matemático}

A equação do calor bidimensional transiente (equação de Fourier) com termo fonte é dada por [2]

$$
\frac{\partial T}{\partial t}=\alpha\left(\frac{\partial^{2} T}{\partial x^{2}}+\frac{\partial^{2} T}{\partial y^{2}}\right)+S(x, y, t)
$$

onde $\alpha$ é a difusividade térmica e $S(x, y, t)$ é o termo fonte. A Eq. (9) fornece a distribuição da temperatura $T$ em domínio retangular, isto é, $\left.\operatorname{com}(x, y) \in \Omega=(0,1) \times(0,1), t \in] 0, t_{f}\right], t_{f}$ indica o tempo final e com condições de contorno de Dirichlet e iniciais dados por

$$
T(0, y, t)=T(L, y, t)=T(x, 0, t)=T(x, L, t)=0
$$

$$
T(x, y, 0)=\operatorname{sen}\left(\frac{\pi x}{L}\right) \operatorname{sen}\left(\frac{\pi y}{L}\right)
$$

e termo fonte dado por

$$
S(x, y, t)=\pi^{2} \operatorname{sen}\left(\frac{\pi x}{L}\right) \operatorname{sen}\left(\frac{\pi y}{L}\right) e^{-\pi^{2} t}
$$

Considerando $L=1 \mathrm{~m}$ e difusividade térmica $\alpha=1 \mathrm{~m}^{2} / \mathrm{s}$, o termo fonte e as condições de contorno foram ajustados por meio do MSF [3] de forma a possibilitar a obtenção da solução analítica dada por

$$
T(x, y, t)=\operatorname{sen}(\pi x) \operatorname{sen}(\pi y) e^{-\pi^{2} t}
$$




\section{Modelo Numérico}

O modelo numérico é caracterizado pelo uso do MDF com malhas uniformes. Com o intuito de verificar as ordens verdadeiras da solução numérica, a priori e a posteriori, o esquema $\theta$ é aplicado, fornecendo assim, a possibilidade de uma análise do comportamento da solução e de seu erro por meio da variação de seu valor. O esquema $\theta$ associa o parâmetro de mistura $(\theta)$ aos esquemas explícito $(\theta=0)$, totalmente implícito $(\theta=1)$ e Crank-Nicolson $\left(\theta=\frac{1}{2}\right)$ [11].

A discretização da equação do calor transiente com termo fonte (equação de Fourier) é pouco discutida na literatura. Em [11], por exemplo, a única referência, se faz ao aplicar o método de Crank-Nicolson em que considera o cálculo da média do termo fonte em níveis de tempo subsequentes. O cálculo do termo fonte pode levar a interpretações equivocadas tanto no tratamento teórico como no tratamento numérico e computacional comprometendo uma análise futura. Nesse sentido, deve-se considerar a expressão

$$
S(x, y, t) \cong \theta S_{i, j, k+1}+(1-\theta) S_{i, j, k}
$$

A discretização da equação do calor bidimensional com termo fonte com erro de truncamento é dada por

$$
\begin{gathered}
T_{t}^{i}-T_{x x}^{i i}-T_{y y}^{i i}-S=-k\left[\frac{1}{2} T_{2 t}^{i i}-\theta\left(T_{2 x t}^{i i i}+T_{y y t}^{i i i}+S_{t}^{i}\right)\right]+T_{4 x}^{i v} \frac{h_{x}^{2}}{12}+T_{4 y}^{i v} \frac{h_{y}^{2}}{12}- \\
k^{2}\left[\frac{1}{6} T_{3 t}^{i i i}-\frac{\theta}{2}\left(T_{2 x 2 t}^{i v}+T_{2 y 2 t}^{i v}+S_{t t}^{i i}\right)\right]-k^{3}\left[\frac{1}{24} T_{4 t}^{i v}-\frac{\theta}{6}\left(T_{2 x 3 t}^{v}+T_{2 y 3 t}^{v}+S_{3 t}^{i i i}\right)\right]+ \\
+T_{6 x}^{v i} \frac{h_{x}^{4}}{360}+T_{6 y}^{v i} \frac{h_{y}^{4}}{360}-k^{4}\left[\frac{1}{120} T_{5 t}^{v}-\frac{\theta}{24}\left(T_{2 x 4 t}^{v i}+T_{2 y 4 t}^{v i}+S_{4 t}^{i v}\right)\right]- \\
-k^{5}\left[\frac{1}{720} T_{6 t}^{v i}-\frac{\theta}{120}\left(T_{2 x 5 t}^{v i i}+T_{2 y 5 t}^{v i i}+S_{5 t}^{v}\right)\right]+T_{8 x}^{v i i i} \frac{h_{x}^{6}}{20160}+T_{8 y}^{v i i i} \frac{h_{y}^{6}}{20160}-\cdots \\
\theta\left(T_{4 x t}^{v} \frac{k h_{x}^{2}}{12}+T_{4 y t}^{v} \frac{k h_{y}^{2}}{12}+T_{4 x 2 t}^{v i} \frac{k^{2} h_{x}^{2}}{24}+T_{4 y 2 t}^{v i} \frac{k^{2} h_{y}^{2}}{24}+T_{4 x 3 t}^{v i i} \frac{k^{3} h_{x}^{2}}{72}+T_{4 y 3 t}^{v i i} \frac{k^{3} h_{y}^{2}}{72}+\right. \\
\left.T_{6 x t}^{v i i} \frac{k h_{x}^{4}}{360}+T_{6 y t}^{v i i} \frac{k h_{y}^{4}}{360}+T_{6 x 2 t}^{v i i i} \frac{k^{2} h_{x}^{4}}{720}+T_{6 y 2 t}^{v i i i} \frac{k^{2} h_{y}^{4}}{720}+T_{t}^{i} 2 \frac{k}{h_{x}^{2}}+T_{t}^{i} 2 \frac{k}{h_{y}^{2}}+T_{2 t}^{i i} \frac{k^{2}}{h_{x}^{2}}+\cdots\right)+\cdots
\end{gathered}
$$

onde a notação $T_{x t}^{i i}$, por exemplo, indica que a função é derivada duas vezes, uma vez na direção $x$ e outra na direção $t$. Os parâmetros $h_{x}$ e $h_{y}$ referem-se ao espaçamento da malha na direção $x$ e $y$, respectivamente e $k$ se refere ao passo no tempo.

Com base na Eq. (15), da mesma forma como no caso unidimensional, pode-se concluir que o erro de truncamento, independente de $\theta$, tem ordem de acurácia $\mathrm{O}\left(h_{x}^{2}+h_{y}^{2}+k\right)$. Porém, verificando atentamente, nota-se que a primeira parcela do lado direito da igualdade é cancelada quando $\theta=\frac{1}{2}$. Portanto, quando $\theta=\frac{1}{2}$ a ordem de acurácia (ou assintótica) é $\mathrm{O}\left(h_{x}^{2}+h_{y}^{2}+k^{2}\right)$, como esperado.

\section{Resultados e Conclusão}

As ordens de acurácia obtidas a posteriori com MER trazem grandes vantagens em relação à perspectiva do comportamento da solução numérica de um problema físico em estudo. Estas podem indicar efetivamente as ordens do erro, uma vez que as ordens de erro a priori devem ser confirmadas pelas ordens obtidas a posteriori. Esta ideia pode ser ampliada para a verificação da solução dos casos em que não há conclusões significativas na estimativa a priori ou a solução analítica é desconhecida. 
A metodologia proposta foi aplicada a diversas variáveis de interesse, a saber, a temperatura no centro do domínio $\left(T_{\mathrm{c}}\right)$, a temperatura média $\left(T_{m}\right)$, a taxa de transferência de calor ao leste $\left(q_{e}\right)$ e a média da norma $l_{1}$.

Resultados a priori e a posteriori são apresentados para a variável $T_{c}=T\left(\frac{1}{2}, \frac{1}{2}\right)$ na Tabela 1 . Como observado na Eq. (15) não se consegue identificar uma sequência, a priori, para as ordens verdadeiras, pode-se concluir somente o valor da ordem assintótica.

\begin{tabular}{ccc}
\hline $\begin{array}{c}\text { Variável de } \\
\text { interesse }\end{array}$ & $\begin{array}{c}\text { Ordens verdadeiras } \\
\text { a priori }\end{array}$ & $\begin{array}{c}\text { Ordens verdadeiras } \\
\text { a posteriori }\end{array}$ \\
\hline \multirow{2}{*}{$T_{c}$} & $\theta=1 \Rightarrow p_{V}=1$ & $\theta=1 \Longrightarrow p_{V}=1,2,3,4, \ldots$ \\
& $\theta=\frac{1}{2} \Rightarrow p_{V}=2$ & $\theta=\frac{1}{2} \Rightarrow p_{V}=2,4,6,8, \ldots$ \\
& $p_{V}$ para $V=2,3,4, \ldots$ não identificado & \\
\hline
\end{tabular}

Tabela 1 Ordens verdadeiras obtidas a priori e a posteriori das aproximações para a equação de Fourier $2 \mathrm{D}$ e para a variável $T_{c}$.

Os resultados obtidos a posteriori podem ser verificados na Figura 1 os quais apresentam as três primeiras ordens verdadeiras obtidas com o emprego de MER para a variável $T_{\mathrm{c}}=T\left(\frac{1}{2}, \frac{1}{2}\right)$ quando $\theta=1$ e $\theta=\frac{1}{2}$.

O método de Crank-Nicolson é comumente empregado em diversas aplicações, confiando seu resultado somente ao conhecimento teórico da ordem assintótica. Verificou-se que a princípio só a ordem assintótica pode ser corroborada a priori, as outras ordens verdadeiras ainda não se pode afirmar corretamente a sua prioridade devido à aproximação de derivadas mistas envolvendo espaço e tempo. Com o emprego de MER o resultado de sua aplicação pode ser comprovado e, além disso, verificado seu comportamento com a redução sistemática da malha. Isto evita, por exemplo, custo computacional desnecessário.

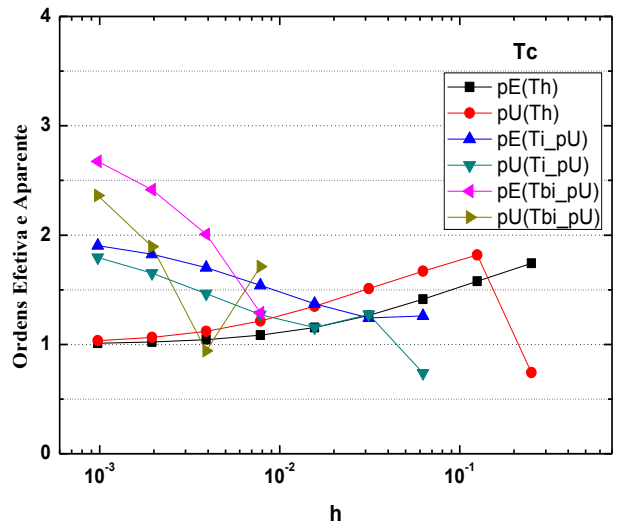

(a) $\theta=1$

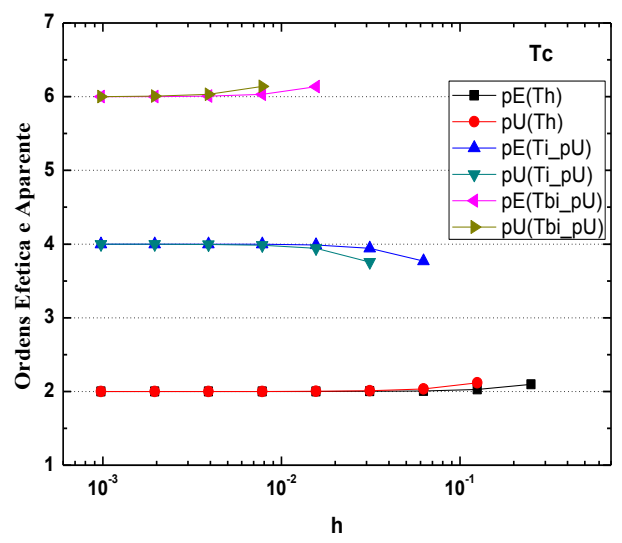

(b) $\theta=\frac{1}{2}$

Figura 1: Ordens verdadeiras - efetiva e aparente da variável $T_{\mathrm{c}}$

A Figura 2 mostra o módulo do erro de discretização com MER (Em1) e sem MER (Eh) para a variável $T_{c}$. Considerando as ordens de magnitude dos erros, pode-se perceber que em geral, para $h \rightarrow 0, E h\left(\theta=\frac{1}{2}\right)<E h(\theta=1)$ e $\operatorname{Em} 1\left(\theta=\frac{1}{2}\right)<E m 1(\theta=1)$, como era esperado. Conclui-se, portanto, que a eficiência de MER não é afetada pelo parâmetro $\theta$ utilizado.

Com a aplicação de MER a verificação do valor correto da ordem assintótica e de ordens verdadeiras do erro de discretização contribui para melhorar a qualidade (acurácia e confiabilidade) das soluções e das estimativas do erro de discretização proporcionando a utilização adequada de esquemas híbridos e parâmetros numéricos em CFD. 


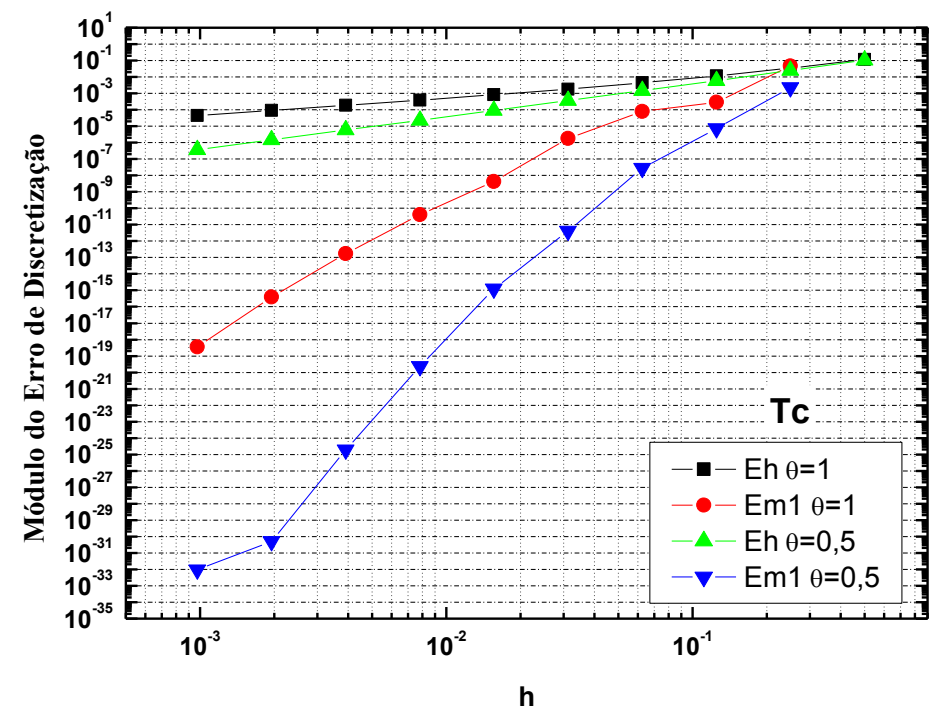

Figura 2: Módulo do erro de discretização com MER (Em1) e sem MER (Eh) para a variável $T_{c}$

\section{Agradecimentos}

Os autores agradecem o apoio financeiro do CNPq (Conselho Nacional de Desenvolvimento Científico e Tecnológico), AEB (Agência Espacial Brasileira) através do Programa Uniespaço, Fundação Araucária (Paraná) e CAPES (Coordenação de Aperfeiçoamento de Pessoal de Nível Superior). O segundo autor é bolsista do CNPq.

\section{Referências}

[1] I. Celik, W. M. Zhang, Calculation of numerical uncertainty using Richardson extrapolation: Application to Some Simple Turbulent Flow Calculations. Journal of Fluids Engineering, vol. 117, n⿳3, pp. 439-445, (1995).

[2] F. P. Incropera, D. P. Dewitt, T. L. Bergman, A. S. Lavine, "Fundamentos de Transferência de Calor e de Massa". Rio de Janeiro: LTC, 2008.

[3] P. M. Knupp, K. Salari, "Verification of computer codes in computational science and engineering". Chapman \& Hall/CRC, 2003.

[4] C. H. Marchi, "Verificação de soluções numéricas unidimensionais em dinâmica dos fluidos". Tese de doutorado, PGMEC-UFSC, Florianópolis, 2001.

[5] C. H. Marchi, L. A. Novak, C. D. Santiago, A. P. S. Vargas, Highly accurate numerical solutions with repeated Richardson extrapolation for CHT and CFD. Journal of Computational and Applied Mathematics. Elsevier, vol 37, pp. 7386-7397 (2013).

[6] W. L. Oberkampf, T. G. Trucano, Verification and validation in computational fluid dynamics. Progress in Aerospace Sciences, vol. 38, pp. 209-272, (2002).

[7] L. F. Richardson, The approximate arithmetical solution by finite differences of physical problems involving differential equations, with an application to the stresses in a masonry dam. Phylosophical Proceedings of the Royal Society of London Serial A, vol. 210, p. 307-357, 1910. [8] P. J. Roache, Perspective: a method for uniform reporting of grid refinement studies. ASME Journal of Fluids Engineering, vol. 116, p. 405-413, (1994).

[9] J. C. Roy. Grid Convergence error analysis for mixed-order numerical schemes. AIAA Journal, vol. 41, no 4, pp. 595-604, (2003).

[10] J. C. Roy. Review of code and solution verification procedures for computational simulation. J. Comp. Phys, vol. 205, p. 131 - 156, (2005).

[11] J. C. Strikwerda, "Finite difference schemes and partial differential equations". 2a ed., Philadelphia, SIAM, 2004. 